\title{
Successful placement of a fully covered esophageal stent to bridge a difficult-to-close mucosal incision during peroral endoscopic myotomy
}
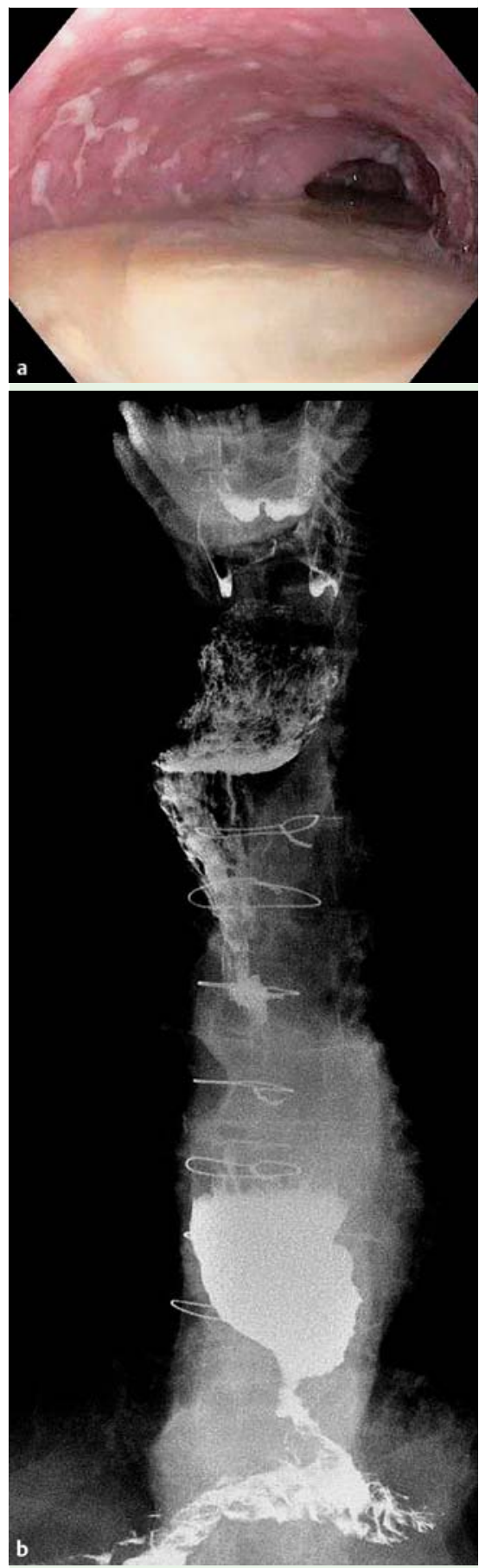

Fig. 1 Achalasia with a dilated esophagus. a Esophagogastroduodenoscopy revealed food retention in a dilated esophagus. b Timed barium esophagram showed delayed esophageal emptying of oral contrast at 10 minutes in the severely dilated tortuous esophagus.

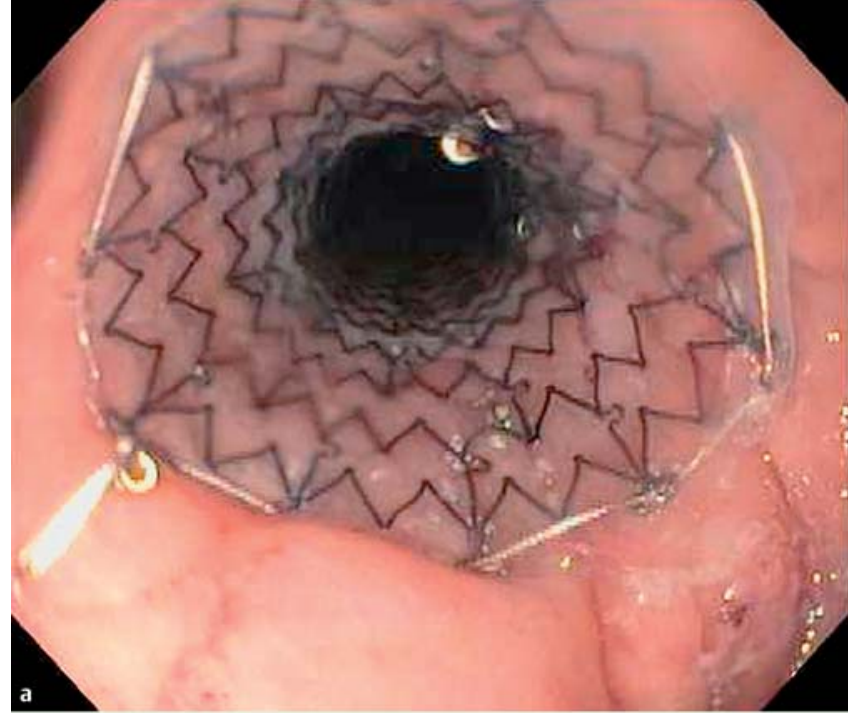

Fig. 2 Stent placement. a Successful deployment of the fully covered esophageal stent completely bridged the mucosal incision. b Chest radiograph on postoperative Day 1 revealed appropriate placement of the esophageal stent in the mid-esophagus.

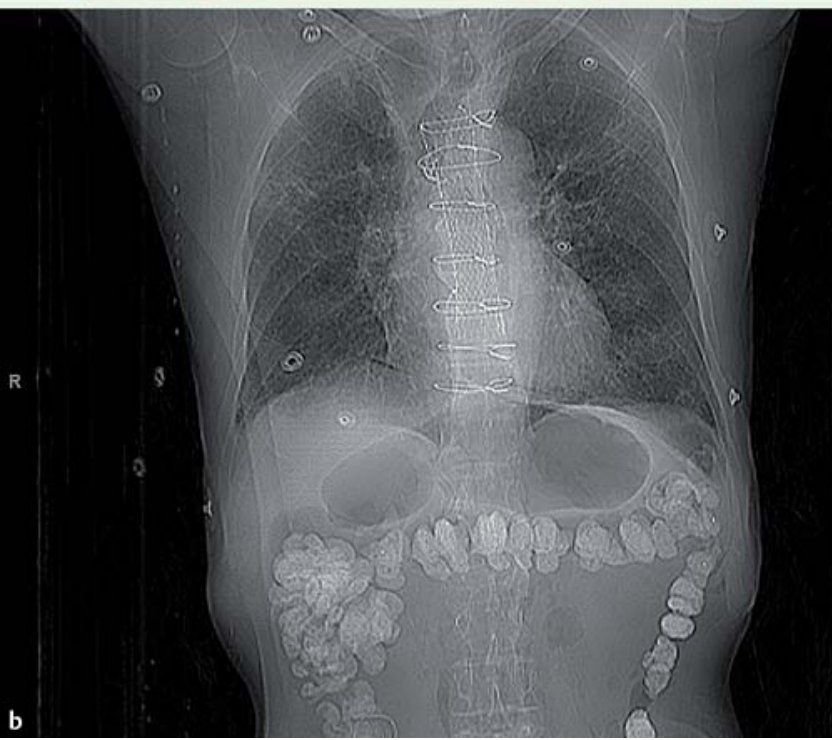

Closure of the mucosal entry during peroral endoscopic myotomy (POEM) can be achieved using standard clips in most cases, but on rare occasions clip closure may not be possible. Alternative salvage methods for closure have been described, including the over-the-scope clip [1,2] and an endoluminal suturing device [3]. We report the first POEM case in which a fully covered stent was used to seal a mucosal incision that was difficult to close.

An 83-year-old man with severe achalasia (Eckardt 9) [4] underwent POEM as described previously [5]. The esophagus was severely dilated and the mucosa was inflamed, presumably from food stasis ( $\bullet$ Fig. 1). The inverted, friable, and edematous edges of the mucosal incision prevented adequate apposition, despite several attempts at closure using different standard clips (Resolution Clip, Boston Scientific, Natick, Massachusetts, United States; Instinct Clip, Cook Endoscopy, Winston-Salem, North Carolina, United States). In total, placement of 18 clips was attempted without success.

The clips were removed and an over-thescope clip (Ovesco Endoscopy AG, Tübingen, Germany) was deployed, approximating three-quarters of the incision. How- 


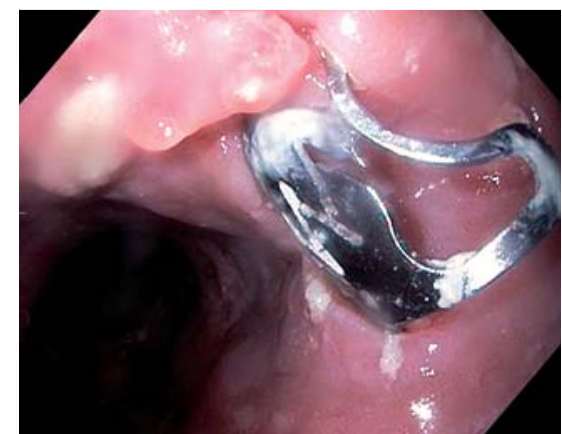

Fig. 3 Esophagogastroduodenoscopy following stent removal. The mucosal entry had healed, with granulation tissue present. The previously placed over-the-scope clip was seen; this was removed at the end of the procedure.

ever, given the persistent mucosal breach, a fully covered $23 \times 120-\mathrm{mm}$ esophageal stent (EndoMaxx; Merit Medical Endotek, South Jordan, Utah, United States) was placed to seal the remaining gap ( Fig.2). Complete healing of the incision was noted on repeat esophagogastroduodenoscopy for stent removal 6 weeks following the procedure ( $\bullet$ Fig. 3 ).

Stent placement for distal esophageal mucosal perforation has been described in a pediatric POEM case [6], but this is the first report on the successful use of a covered esophageal stent to bridge a difficult-to-close mucosal incision. By successfully closing the incision with the stent, the serious complications associated with leakage of esophageal contents into the mediastinum and potential surgery were prevented. Esophageal stenting is feasible and should be considered as a salvage method for closure of mucosal entry incision in POEM.

Endoscopy_UCTN_Code_TTT_1AO_2AZ

Competing interests: None

\section{Dennis Yang, Qing Zhang, Peter V. Draganov}

Division of Gastroenterology, University of Florida, Gainesville, Florida, United States

\section{References}

1 Saxena P, Chavez YH, Kord Valeshabad A et al. An alternative method for mucosal flap closure during peroral endoscopic myotomy using an over-the-scope clipping device. Endoscopy 2013; 45: 579-581

2 Yang D, Draganov PV. Closing the gap in POEM. Endoscopy 2013; 45: 677

3 Kurian AA, Bhayani NH, Reavis K et al. Endoscopic suture repair of full-thickness esoph- agotomy during per-oral esophageal myotomy for achalasia. Surg Endosc 2013; 27: 3910

4 Eckardt VF. Clinical presentations and complications of achalasia. Gastrointest Endosc Clin N Am 2001; 11: 281-292

5 Inoue H, Minami H, Kobayashi Yet al. Peroral endoscopic myotomy (POEM) for esophageal achalasia. Endoscopy 2010; 42: $265-$ 271

6 Ling T, Pei Q Pan J et al. Successful use of a covered, retrievable stent to seal a ruptured mucosal flap safety valve during peroral endoscopic myotomy in a child with achalasia. Endoscopy 2013; 45 (Suppl. 02): E6364

\section{Bibliography}

Dol http://dx.doi.org/

10.1055/s-0034-1377547

Endoscopy 2014; 46: E467-E468

(c) Georg Thieme Verlag KG

Stuttgart · New York

ISSN 0013-726X

\section{Corresponding author \\ Peter V. Draganov, MD}

Division of Gastroenterology

University of Florida

1600 SW Archer Road, Room HD 602

Gainesville, FL 32610

United States

Fax: +1-352-392-3618

peter.draganov@medicine.ufl.edu 